\title{
Stability and Superstability of Generalized $(\theta, \phi)$-Derivations in Non-Archimedean Algebras: Fixed Point Theorem via the Additive Cauchy Functional Equation
}

\author{
M. Eshaghi Gordji, ${ }^{1}$ M. B. Ghaemi, ${ }^{2}$ G. H. Kim, ${ }^{3}$ \\ and Badrkhan Alizadeh ${ }^{4}$ \\ ${ }^{1}$ Department of Mathematics, Semnan University, P.O. Box 35195-363, Semnan, Iran \\ ${ }^{2}$ Department of Mathematics, Iran University of Science and Technology, Narmak, Tehran, Iran \\ ${ }^{3}$ Department of Mathematics, Kangnam University, Yongin, Gyeonggi 446-702, Republic of Korea \\ ${ }^{4}$ Technical and Vocational Faculty of Tabriz, Technical and Vocational University of Iran, \\ P.O. Box 51745-135, Tabriz, Iran
}

Correspondence should be addressed to G. H. Kim, ghkim@kangnam.ac.kr

Received 23 September 2011; Revised 6 October 2011; Accepted 16 October 2011

Academic Editor: Ferenc Hartung

Copyright (c) 2011 M. Eshaghi Gordji et al. This is an open access article distributed under the Creative Commons Attribution License, which permits unrestricted use, distribution, and reproduction in any medium, provided the original work is properly cited.

Let $A$ be an algebra, and let $\theta, \phi$ be ring automorphisms of $A$. An additive mapping $H: A \rightarrow A$ is called a $(\theta, \phi)$-derivation if $H(x y)=H(x) \theta(y)+\phi(x) H(y)$ for all $x, y \in A$. Moreover, an additive mapping $F: A \rightarrow A$ is said to be a generalized $(\theta, \phi)$-derivation if there exists a $(\theta, \phi)$ derivation $H: A \rightarrow A$ such that $F(x y)=F(x) \theta(y)+\phi(x) H(y)$ for all $x, y \in A$. In this paper, we investigate the superstability of generalized $(\theta, \phi)$-derivations in non-Archimedean algebras by using a version of fixed point theorem via Cauchy's functional equation.

\section{Introduction and Preliminaries}

In 1897, Hensel [1] has introduced a normed space which does not have the Archimedean property. It turned out that non-Archimedean spaces have many nice applications [2,3].

A non-Archimedean field is a field $\mathbb{K}$ equipped with a function (valuation) $|\cdot|$ from $\mathbb{K}$ into $[0, \infty)$ such that $|r|=0$ if and only if $r=0,|r s|=|r||s|$, and $|r+s| \leq \max \{|r|,|s|\}$ for all $r, s \in \mathbb{K}$. An example of a non-Archimedean valuation is the mapping $|\cdot|$ taking everything but 0 into 1 and $|0|=0$. This valuation is called trivial (see [4]). 
Definition 1.1. Let $X$ be a vector space over a scalar field $\mathbb{K}$ with a non-Archimedean nontrivial valuation $|\cdot|$. A function $\|\cdot\|: X \rightarrow \mathbb{R}$ is a non-Archimedean norm (valuation) if it satisfies the following conditions:

$\left(\mathrm{NA}_{1}\right)\|x\|=0$ if and only if $x=0$,

$\left(\mathrm{NA}_{2}\right)\|r x\|=|r|\|x\|$ for all $r \in \mathbb{K}$ and $x \in X$,

$\left(\mathrm{NA}_{3}\right)\|x+y\| \leq \max \{\|x\|,\|y\|\}$ for all $x, y \in X$ (the strong triangle inequality).

A sequence $\left\{x_{m}\right\}$ in a non-Archimedean space is Cauchy's if and only if $\left\{x_{m+1}-x_{m}\right\}$ converges to zero. By a complete non-Archimedean space, we mean one in which every Cauchy's sequence is convergent. A non-Archimedean-normed algebra is a non-Archimedean-normed space $A$ with a linear associative multiplication, satisfying $\|x y\| \leq\|x\|\|y\|$ for all $x, y \in A$. A non-Archimedean complete normed algebra is called a non-Archimedean Banach's algebra (see [5]).

Definition 1.2. Let $X$ be a nonempty set, and let $d: X \times X \rightarrow[0, \infty]$ satisfy the following properties:

$\left(\mathrm{D}_{1}\right) d(x, y)=0$ if and only if $x=y$,

$\left(\mathrm{D}_{2}\right) d(x, y)=d(y, x)$ (symmetry),

$\left(\mathrm{D}_{3}\right) d(x, z) \leq \max \{d(x, y), d(y, z)\}$ (strong triangle inequality),

for all $x, y, z \in X$. Then $(X, d)$ is called a non-Archimedean generalized metric space. $(X, d)$ is called complete if every $d$-Cauchy's sequence in $X$ is $d$-convergent.

Definition 1.3. Let $A$ be a non-Archimedean algebra, and let $\theta, \phi$ be ring automorphisms of $A$. An additive mapping $H: A \rightarrow A$ is called a $(\theta, \phi)$-derivation in case $H(x y)=H(x) \theta(y)+$ $\phi(x) H(y)$ holds for all $x, y \in A$. An additive mapping $F: A \rightarrow A$ is said to be a generalized $(\theta, \phi)$-derivation if there exists a $(\theta, \phi)$-derivation $H: A \rightarrow A$ such that

$$
F(x y)=F(x) \theta(y)+\phi(x) H(y)
$$

for all $x, y \in A$.

We need the following fixed point theorem (see $[6,7])$.

Theorem 1.4 (Non-Archimedean Alternative Contraction Principle). Suppose $(X, d)$ is a nonArchimedean generalized complete metric space and $\Lambda: X \rightarrow X$ is a strictly contractive mapping; that is,

$$
d(\Lambda x, \Lambda y) \leq L d(x, y) \quad(x, y \in X)
$$

for some $L<1$. If there exists a nonnegative integer $k$ such that $d\left(\Lambda^{k+1} x, \Lambda^{k} x\right)<\infty$ for some $x \in X$, then the followings are true.

(a) The sequence $\left\{\Lambda^{n} x\right\}$ converges to a fixed point $x^{*}$ of $\Lambda$.

(b) $x^{*}$ is a unique fixed point of $\Lambda$ in

$$
X^{*}=\left\{y \in X \mid d\left(\Lambda^{k} x, y\right)<\infty\right\}
$$


(c) If $y \in X^{*}$, then

$$
d\left(y, x^{*}\right) \leq d(\Lambda y, y)
$$

A functional equation $(\xi)$ is superstable if every approximately solution of $(\xi)$ is an exact solution of it.

The stability of functional equations was first introduced by Ulam [8] during his talk before a mathematical colloquium at the University of Wisconsin in 1940. In 1941, Hyers [9] gave a first affirmative answer to the question of Ulam for Banach spaces. In 1978, Rassias [10] generalized the theorem of Hyers by considering the stability problem with unbounded Cauchy's differences $\|f(x+y)-f(x)-f(y)\| \leq \epsilon\left(\|x\|^{p}+\|y\|^{p}\right),(\epsilon>0, p \in[0,1))$. Moreover, John Rassias [11-13] investigated the stability of some functional equations when the control function is the product of powers of norms. In 1991, Gajda [14] answered the question for the case $p>1$, which was raised by Rassias. This new concept is known as the Hyers-UlamRassias or the generalized Hyers-Ulam stability of functional equations ([11-13, 15-35]).

In 1992, Găvruţa [36] generalized the Rassias theorem as follows.

Suppose $(G,+)$ is an ablian group, $X$ is a Banach space, $\varphi: G \times G \rightarrow[0, \infty)$ satisfies

$$
\tilde{\varphi}(x, y)=\frac{1}{2} \sum_{n=0}^{\infty} 2^{-n} \varphi\left(2^{n} x, 2^{n} y\right)<\infty,
$$

for all $x, y \in G$. If $f: G \rightarrow X$ is a mapping with

$$
\|f(x+y)-f(x)-f(y)\| \leq \varphi(x, y),
$$

for all $x, y \in G$, then there exists a unique mapping $T: G \rightarrow X$ such that $T(x+y)=T(x)+T(y)$ and $\|f(x)-T(x)\| \leq \tilde{\varphi}(x, x)$ for all $x, y \in G$.

In 1949, Bourgin [37] proved the following result, which is sometimes called the superstability of ring homomorphisms: suppose that $A$ and $B$ are Banach algebras with unit. If $f: A \rightarrow B$ is a surjective mapping such that

$$
\begin{aligned}
& \|f(x+y)-f(x)-f(y)\| \leq \epsilon, \\
& \|f(x y)-f(x) f(y)\| \leq \delta,
\end{aligned}
$$

for some $\epsilon \geq 0, \delta \geq 0$ and for all $x, y \in A$, then $f$ is a ring homomorphism.

The first superstability result concerning derivations between operator algebras was obtained by Šemrl in [38]. Badora [39] proved the superstability of the functional equation $f(x y)=x f(y)+f(x) y$, where $f$ is a mapping on normed algebra $A$ with unit. Ansari-Piri and Anjidani [40] discussed the superstability of generalized derivations on Banach's algebras. Recently, Eshaghi Gordji et al. [41] investigated the stability and superstability of higher ring derivations on non-Archimedean Banach's algebras (see also [42]). In this paper, we investigate the superstability of generalized $(\theta, \phi)$-derivations on non-Archimedean Banach algebras by using the fixed point methods. 


\section{Non-Archimedean Superstability of Generalized $(\theta, \phi)$-Derivations}

In this paper, we assume that $A$ is a non-Archimedean Banach's algebra, with unit over a non-Archimedean field $\mathbb{K}$, and $\theta, \phi$ are ring automorphisms of $A$.

Theorem 2.1. Let $\varphi, \psi: A \times A \rightarrow[0, \infty)$ be functions. Suppose that $f: A \rightarrow A$ is a mapping such that

$$
\begin{gathered}
\|f(x+y)-f(x)-f(y)\| \leq \varphi(x, y), \\
\|f(x y)-f(x) \theta(y)-\phi(x) g(y)\| \leq \psi(x, y),
\end{gathered}
$$

for all $x, y \in A$. If there exist constants $K, L<1$ and a natural number $k \in \mathbb{K}$,

$$
|k|^{-1} \varphi(k x, k y) \leq L \varphi(x, y), \quad|k|^{-1} \psi(k x, y), \quad|k|^{-1} \psi(x, k y) \leq K \psi(x, y),
$$

for all $x, y \in A$, then $f$ is a generalized $(\theta, \phi)$-derivation and $g$ is a $(\theta, \phi)$-derivation.

Proof. By induction on $i$, we prove that for each $n \in \mathbb{N}_{0}$, for all $x \in A$ and $i \geq 2$,

$$
\|f(i x)-i f(x)\| \leq \max \{\varphi(0,0), \varphi(x, x), \varphi(2 x, x), \ldots, \varphi((i-1) x, x)\} .
$$

Let $x=y$ in (2.1), then

$$
\|f(2 x)-2 f(x)\| \leq \max \{\varphi(0,0), \varphi(x, x)\}, \quad n \in \mathbb{N}_{0}, x \in A .
$$

This proves (2.4) for $i=2$. Let (2.4) hold for $i=1,2, \ldots, J$. Replacing $x$ by $j x$ and $y$ by $x$ in (2.1) for each $n \in \mathbb{N}_{0}$, and for all $x \in A$, we get

$$
\|f((j+1) x)-f(j x)-f(x)\| \leq \max \{\varphi(0,0), \varphi(j x, x)\} .
$$

Since

$$
\begin{aligned}
f((j+1) x)-f(j x)-f(x) & =f((j+1) x)-(j+1) f(x)+(j+1) f(x)-f(j x)-f(x) \\
& =f((j+1) x)-(j+1) f(x)+j f(x)-f(j x),
\end{aligned}
$$

for all $x \in A$, it follows from induction hypothesis and (2.6) that, for all $x \in A$,

$$
\begin{aligned}
\|f((j+1) x)-(j+1) f(x)\| & \leq \max \{\|f((j+1) x)-f(j x)-f(x)\|,\|j f(x)-f(j x)\|\} \\
& \leq \max \{\varphi(0,0), \varphi(x, x), \varphi(2 x, x), \ldots, \varphi((j) x, x)\} .
\end{aligned}
$$


This proves (2.4) for all $i \geq 2$. In particular, for all $x \in A$,

$$
\|f(k x)-k f(x)\| \leq \Phi(x)
$$

where

$$
\Phi(x)=\max \{\varphi(0,0), \varphi(x, x), \varphi(2 x, x), \ldots, \varphi((k-1) x, x)\} \quad(x \in A)
$$

Let us define a set $X$ of all functions $r: A \rightarrow A$ by

$$
X=\{r: A \longrightarrow A\}
$$

and introduce $d$ on $X$ as follows:

$$
d(r, s)=\inf \{\alpha>0:\|r(x)-s(x)\| \leq \alpha \Phi(x) \forall x \in A\} .
$$

It is easy to see that $d$ defines a generalized complete metric on $X$. Define $J: X \rightarrow X$ by $J(r)(x)=k^{-1} r(k x)$. Then $J$ is strictly contractive on $X$, in fact if

$$
\|r(x)-s(x)\| \leq \alpha \Phi(x) \quad(x \in A)
$$

then, by (2.3),

$$
\|J(r)(x)-J(s)(x)\|=|k|^{-1}\|r(k x)-s(k x)\| \leq \alpha|k|^{-1} \Phi(k x) \leq L \alpha \Phi(x) \quad(x \in A) .
$$

It follows that

$$
d(J(r), J(s)) \leq L d(r, s) \quad(g, h \in X)
$$

Hence, $J$ is strictly contractive mapping with the Lipschitz constant L. By (2.9),

$$
\begin{aligned}
& \|(J f)(x)-f(x)\|=\left\|k^{-1} f(k x)-f(x)\right\|, \\
& |k|^{-1}\|f(k x)-k f(x)\| \leq|k|^{-1} \Phi(x) \quad(x \in A) .
\end{aligned}
$$

This means that $d(J(f), f) \leq 1 /|k|$. By Theorem 1.4, $J$ has a unique fixed point $h: A \rightarrow A$ in the set

$$
U=\{r \in X: d(r, J(f))<\infty\}
$$

and, for each $x \in A$,

$$
h(x)=\lim _{m \rightarrow \infty} J^{m}(f(x))=\lim k^{-m} f\left(k^{m} x\right)
$$


Therefore, each $x, y \in A$,

$$
\begin{aligned}
\|h(x+y)-h(x)-h(y)\| & =\lim _{m \rightarrow \infty}|k|^{-m}\left\|f\left(k^{m}(x+y)\right)-f\left(k^{m} x\right)-f\left(k^{m} y\right)\right\| \\
& \leq \lim _{m \rightarrow \infty}|k|^{-m} \max \left\{\varphi(0,0), \varphi\left(k^{n} x, k^{n} y\right)\right\} \\
& \leq \lim _{m \rightarrow \infty} L^{m} \varphi(x, y)=0 .
\end{aligned}
$$

This shows that $h$ is additive.

Replacing $y$ by $k^{n} y$ in (2.2), we get

$$
\left\|f\left(k^{n} x y\right)-f(x) \theta\left(k^{n} y\right)-\phi(x) g\left(k^{n} y\right)\right\| \leq \psi\left(x, k^{n} y\right),
$$

and so

$$
\left\|\frac{f\left(k^{n} x y\right)}{k^{n}}-f(x) \theta(y)-\phi(x) \frac{g\left(k^{n} y\right)}{k^{n}}\right\| \leq \frac{1}{|k|^{n}} \psi\left(x, k^{n} y\right) \leq K^{n} \psi(x, y),
$$

for all $x, y \in A$ and each $n \in \mathbb{N}$. By taking $n \rightarrow \infty$, we have

$$
h(x y)=f(x) \theta(y)+\lim _{n \rightarrow \infty} \phi(x) \frac{g\left(k^{n} y\right)}{k^{n}}
$$

for all $x, y \in A$.

Fix $m \in \mathbb{N}$. By (2.22), we have

$$
\begin{aligned}
f\left(k^{m} x\right) \theta(y) & =h\left(k^{m} x y\right)-\lim _{n \rightarrow \infty} \phi\left(k^{m} x\right)\left(\frac{g\left(k^{n} y\right)}{k^{n}}\right) \\
& =f(x) \theta\left(k^{m} y\right)+\lim _{n \rightarrow \infty} \phi(x)\left(\frac{g\left(k^{n} k^{m} x\right)}{k^{n}}\right)-k^{m} \lim _{n \rightarrow \infty} \phi(x)\left(\frac{g\left(k^{n} x\right)}{k^{n}}\right) \\
& =k^{m} f(x) \theta(y)+k^{m} \lim _{n \rightarrow \infty} \phi(x)\left(\frac{g\left(k^{n+m} x\right)}{k^{n+m}}\right)-k^{m} \lim _{n \rightarrow \infty} \phi(x)\left(\frac{g\left(k^{n} x\right)}{k^{n}}\right) \\
& =k^{m} f(x) \theta(y),
\end{aligned}
$$

for all $x, y \in A$. Then $f(x) \theta(y)=\left(f\left(k^{m} x\right) / k^{m}\right) \theta(y)$ for all $x, y \in A$ and each $m \in \mathbb{N}$, and so, by taking $m \rightarrow \infty$, we have $f(x) \theta(y)=h(x) \theta(x)$. Now we obtain $h=f$, since $A$ is with unit. Replacing $x$ by $k^{n} x$ in (2.2), we obtain

$$
\left\|f\left(k^{n}(x y)\right)-f\left(k^{n} x\right) \theta(y)-\phi\left(k^{n} x\right) g(y)\right\| \leq \psi\left(k^{n} x, y\right),
$$


and; hence,

$$
\left\|\frac{f\left(k^{n} x y\right)}{k^{n}}-\frac{f\left(k^{n} x\right)}{k^{n}} \theta(y)-\phi(x) g(y)\right\| \leq \frac{1}{|k|^{n}} \psi\left(k^{n} x, y\right) \leq K^{n} \psi(x, y),
$$

for all $x, y \in A$ and each $n \in \mathbb{N}$. Sending $n$ to infinite, we have

$$
f(x y)=f(x) \theta(y)+\phi(x) g(y)
$$

By (2.26), we get

$$
\begin{aligned}
\phi(z) g(x y) & =f(z x y)-f(z) \theta(x y) \\
& =f(z x) \theta(y)+\phi(z x) g(y)-f(z) \theta(x y) \\
& =[f(z) \theta(x)+\phi(z) g(x)] \theta(y)+\phi(z x) g(y)-f(z) \theta(x y) \\
& =\phi(z)[g(x) \theta(y)+\phi(x) g(y)],
\end{aligned}
$$

for all $x, y, z \in A$. Therefore, we have $g(x y)=g(x) \theta(y)+\phi(x) g(y)$.

Since $f(x y)=f(x) \theta(y)+\phi(x) g(y), f$ is additive, and $A$ is with unit, $g$ is additive. ted.

The proof of the following theorem is similar to that in Theorem 2.1; hence, it is omit-

Theorem 2.2. Let $\varphi, \psi: A \times A \rightarrow[0, \infty)$ be functions. Suppose that $f: A \rightarrow A$ and $g: A \rightarrow A$ are mappings such that

$$
\begin{gathered}
\|f(x+y)-f(x)-f(y)\| \leq \varphi(x, y), \\
\|f(x y)-x f(y)-g(x) y\| \leq \psi(x, y),
\end{gathered}
$$

for all $x, y \in A$. If there exists constants $K, L<1$ and a natural number $k \in \mathbb{K}$,

$$
|k| \varphi\left(k^{-1} x, k^{-1} y\right) \leq L \varphi(x, y),|k| \psi\left(k^{-1} x, y\right),|k| \psi\left(x, k^{-1} y\right) \leq K \psi(x, y),
$$

for all $x, y \in A$, then $f$ is a generalized $(\theta, \phi)$-derivation and $g$ is a $(\theta, \phi)$-derivation.

In the following corollaries $\mathbb{Q}_{p}$ is the field of $p$-adic numbers.

Corollary 2.3. Let $A$ be a non-Archimedean Banach algebra over $\mathbb{Q}_{p}, \varepsilon>0$, and let $p_{1}, p_{2} \in(1, \infty)$. Suppose that

$$
\begin{aligned}
& \|f(x+y)-f(x)-f(y)\| \leq \varepsilon\left(\|x\|^{p_{1}}\|y\|^{p_{2}}\right), \\
& \|f(x y)-x f(y)-g(x) y\| \leq \varepsilon\left(\|x\|^{p_{1}}\|y\|^{p_{2}}\right),
\end{aligned}
$$

for all $x, y \in A$. Then $f$ is a generalized $(\theta, \phi)$-derivation and $g$ is a $(\theta, \phi)$-derivation. 
Proof. Let $\varphi(x, y)=\psi(x, y)=\varepsilon\left(\|x\|^{p_{1}}\|y\|^{p_{2}}\right)$ for all $x, y \in A$; then

$$
\begin{aligned}
& |p|^{-1} \varphi(p x, p y)=|p|^{p_{1}+p_{2}-1} \varepsilon\left(\|x\|^{p_{1}}\|y\|^{p_{2}}\right), \\
& |p|^{-1} \varphi(p x, y)=|p|^{p_{1}-1} \varepsilon\left(\|x\|^{p_{1}}\|y\|^{p_{2}}\right), \\
& |p|^{-1} \varphi(x, p y)=|p|^{p_{2}-1} \varepsilon\left(\|x\|^{p_{1}}\|y\|^{p_{2}}\right) .
\end{aligned}
$$

Put

$$
\begin{aligned}
L & =K=\max \left\{|p|^{p_{1}-1},|p|^{p_{2}-1},|p|^{p_{1}+p_{2}-1}\right\} \\
& =\max \left\{p^{1-p_{1}}, p^{1-p_{2}}, p^{1-p_{1}-p_{2}}\right\} .
\end{aligned}
$$

So, by Theorem 2.1, $f$ is a generalized $(\theta, \phi)$-derivation and $g$ is a $(\theta, \phi)$-derivation.

Corollary 2.4. Let $A$ be a non-Archimedean Banach algebra over $\mathbb{Q}_{p}, \varepsilon>0$, and let $p_{1}, p_{2}, p_{1}+p_{2} \in$ $(-\infty, 1)$. Suppose that

$$
\begin{aligned}
& \|f(x+y)-f(x)-f(y)\| \leq \varepsilon\left(\|x\|^{p_{1}}\|y\|^{p_{2}}\right), \\
& \|f(x y)-x f(y)-g(x) y\| \leq \varepsilon\left(\|x\|^{p_{1}}\|y\|^{p_{2}}\right),
\end{aligned}
$$

for all $x, y \in A$. Then $f$ is a generalized $(\theta, \phi)$-derivation and $g$ is a $(\theta, \phi)$-derivation.

Proof. Let $\varphi(x, y)=\psi(x, y)=\varepsilon\left(\|x\|^{p_{1}}\|y\|^{p_{2}}\right)$ for all $x, y \in A$, then

$$
\begin{aligned}
& |p| \varphi\left(p^{-1} x, p^{-1} y\right)=|p|^{1-p_{1}-p_{2}} \varepsilon\left(\|x\|^{p_{1}}\|y\|^{p_{2}}\right), \\
& |p| \varphi\left(p^{-1} x, y\right)=|p|^{1-p_{1}} \varepsilon\left(\|x\|^{p_{1}}\|y\|^{p_{2}}\right) \\
& |p| \varphi\left(x, p^{-1} y\right)=|p|^{1-p_{2}} \varepsilon\left(\|x\|^{p_{1}}\|y\|^{p_{2}}\right) .
\end{aligned}
$$

Put

$$
\begin{aligned}
L & =K=\max \left\{|p|^{1-p_{1}},|p|^{1-p_{2}},|p|^{1-p_{1}-p_{2}}\right\} \\
& =\max \left\{p^{p_{1}-1}, p^{p_{2}-1}, p^{p_{1}+p_{2}-1}\right\} .
\end{aligned}
$$

So, by Theorem 2.2, $f$ is a generalized $(\theta, \phi)$-derivation and $g$ is a $(\theta, \phi)$-derivation. 
Similarly, we can obtain the following results.

Corollary 2.5. Let $A$ be a non-Archimedean Banach's algebra over $\mathbb{Q}_{p}, \varepsilon>0, \delta>0$, and let $p_{1}, p_{2} \in$ $(1, \infty)$. Suppose that

$$
\begin{aligned}
& \|f(x+y)-f(x)-f(y)\| \leq \varepsilon\left(\|x\|^{p_{1}}+\|y\|^{p_{2}}\right), \\
& \|f(x y)-x f(y)-g(x) y\| \leq \delta\left(\|x\|^{p_{1}}\|y\|^{p_{2}}\right),
\end{aligned}
$$

for all $x, y \in A$. Then $f$ is a generalized $(\theta, \phi)$-derivation and $g$ is a $(\theta, \phi)$-derivation.

Corollary 2.6. Let $A$ be a non-Archimedean Banach's algebra over $\mathbb{Q}_{p}, \varepsilon>0, \delta>0$, and let $p_{1}, p_{2} \in$ $(1, \infty)$. Suppose that

$$
\begin{aligned}
& \max \{\|f(x+y)-f(x)-f(y)\|,\|f(x y)-x f(y)-g(x) y\|\} \\
& \leq \varepsilon \min \left\{\left(\|x\|^{p_{1}}+\|y\|^{p_{2}}\right),\|x\|^{p_{1}}\|y\|^{p_{2}}\right\}
\end{aligned}
$$

for all $x, y \in A$. Then $f$ is a generalized $(\theta, \phi)$-derivation and $g$ is a $(\theta, \phi)$-derivation.

Corollary 2.7. Let $A$ be a non-Archimedean Banach's algebra over $\mathbb{Q}_{p}, \varepsilon>0, \delta>0$, and let $p_{1}, p_{2}, p_{1}+p_{2} \in(-\infty, 1)$. Suppose that

$$
\begin{aligned}
& \|f(x+y)-f(x)-f(y)\| \leq \varepsilon\left(\|x\|^{p_{1}}+\|y\|^{p_{2}}\right), \\
& \|f(x y)-x f(y)-g(x) y\| \leq \delta\left(\|x\|^{p_{1}}\|y\|^{p_{2}}\right),
\end{aligned}
$$

for all $x, y \in A$. Then $f$ is a generalized $(\theta, \phi)$-derivation and $g$ is a $(\theta, \phi)$-derivation.

\section{Acknowledgment}

The third author of this work was partially supported by Basic Science Research Program through the National Research Foundation of Korea (NRF) funded by the Ministry of Education, Science and Technology (Grant number: 2011-0005197).

\section{References}

[1] K. Hensel, “Uber eine neue Begrundung der Theorie der algebraischen Zahlen,” Jahresber. Deutsch. Math. Verein, vol. 6, pp. 83-88, 1897.

[2] A. Khrennikov, Non-Archimedean analysis: quantum paradoxes, dynamical systems and biological models, vol. 427 of Mathematics and Its Applications, Kluwer Academic Publishers, Dordrecht, The Netherlands, 1997.

[3] A. C. M. van Rooij, Non-Archimedean Functional Analysis, vol. 51 of Monographs and Textbooks in Pure and Applied Math., Marcel Dekker, New York, NY, USA, 1978.

[4] L. M. Arriola and W. A. Beyer, "Stability of the Cauchy functional equation over $p$-adic fields," Real Analysis Exchange, vol. 31, no. 1, pp. 125-132, 2005/06.

[5] J. A. Alvarez, "C $C^{*}$-algebras of operators in non-Archimedean Hilbert spaces," Commentationes Mathematicae Universitatis Carolinae, vol. 33, no. 4, pp. 573-580, 1992. 
[6] A. K. Mirmostafaee, "Non-Archimedean stability of the monomial functional equations," Tamsui Oxford Journal of Mathematical Sciences, vol. 26, no. 2, pp. 221-235, 2010.

[7] A. K. Mirmostafaee, "Non-Archimedean stability of quadratic equations," Fixed Point Theory, vol. 11, no. 1, pp. 67-75, 2010.

[8] S. M. Ulam, Problems in Modern Mathematics, John Wiley \& Sons, New York, NY, USA, 1964.

[9] D. H. Hyers, "On the stability of the linear functional equation," Proceedings of the National Academy of Sciences of the United States of America, vol. 27, pp. 222-224, 1941.

[10] T. M. Rassias, "On the stability of the linear mapping in Banach spaces," Proceedings of the American Mathematical Society, vol. 72, no. 2, pp. 297-300, 1978.

[11] J. M. Rassias, "On approximation of approximately linear mappings by linear mappings," Journal of Functional Analysis, vol. 46, no. 1, pp. 126-130, 1982.

[12] J. M. Rassias, “On approximation of approximately linear mappings by linear mappings,” Bulletin des Sciences Mathématiques. 2e Série, vol. 108, no. 4, pp. 445-446, 1984.

[13] J. M. Rassias, "Solution of a problem of Ulam," Journal of Approximation Theory, vol. 57, no. 3, pp. 268-273, 1989.

[14] Z. Gajda, "On stability of additive mappings," International Journal of Mathematics and Mathematical Sciences, vol. 14, no. 3, pp. 431-434, 1991.

[15] M. A. SiBaha, B. Bouikhalene, and E. Elqorachi, “Ulam-Gavruta-Rassias stability of a linear functional equation," International Journal of Applied Mathematics \& Statistics, vol. 7, no. Fe07, pp. 157-166, 2007.

[16] B. Bouikhalene and E. Elqorachi, "Ulam-Găvruta-Rassias stability of the Pexider functional equation," International Journal of Applied Mathematics E Statistics, vol. 7, no. Fe07, pp. 27-39, 2007.

[17] H.-X. Cao, J.-R. Lv, and J. M. Rassias, "Superstability for generalized module left derivations and generalized module derivations on a Banach module. I," Journal of Inequalities and Applications, vol. 2009, Article ID 718020, 10 pages, 2009.

[18] H.-X. Cao, J.-R. Lv, and J. M. Rassias, "Superstability for generalized module left derivations and generalized module derivations on a Banach module. II," Journal of Inequalities in Pure and Applied Mathematics, vol. 10, no. 3, article 85, 2009.

[19] M. Eshaghi Gordji, A. Ebadian, and S. Zolfaghari, "Stability of a functional equation deriving from cubic and quartic functions," Abstract and Applied Analysis, vol. 2008, Article ID 801904, 17 pages, 2008.

[20] M. Eshaghi Gordji, S. Kaboli Gharetapeh, J. M. Rassias, and S. Zolfaghari, "Solution and stability of a mixed type additive, quadratic, and cubic functional equation," Advances in Difference Equations, vol. 2009, Article ID 826130, 17 pages, 2009.

[21] M. Eshaghi Gordji and H. Khodaei, "On the generalized Hyers-Ulam-Rassias stability of quadratic functional equations," Abstract and Applied Analysis, vol. 2009, Article ID 923476, 11 pages, 2009.

[22] M. Eshaghi Gordji, H. Khodaei, and R. Khodabakhsh, "General quartic-cubic-quadratic functional equation in non-Archimedean normed spaces," "Politehnica" University of Bucharest. Scientific Bulletin. Series A, vol. 72, no. 3, pp. 69-84, 2010.

[23] M. Eshaghi Gordji and M. B. Savadkouhi, "Stability of mixed type cubic and quartic functional equations in random normed spaces," Journal of Inequalities and Applications, vol. 2009, Article ID 527462, 9 pages, 2009.

[24] M. Eshaghi Gordji, S. Zolfaghari, J. M. Rassias, and M. B. Savadkouhi, "Solution and stability of a mixed type cubic and quartic functional equation in quasi-Banach spaces," Abstract and Applied Analysis, vol. 2009, Article ID 417473, 14 pages, 2009.

[25] M. Eshaghi Gordji and H. Khodaei, Stability of Functional Equations, LAP LAMBERT Academic Publishing, 2010.

[26] R. Farokhzad and S. A. R. Hosseinioun, "Perturbations of Jordan higher derivations in Banach ternary algebras: an alternative fixed point approach," International Journal of Nonlinear Analysis and Applications, vol. 1, no. 1, pp. 42-53, 2010.

[27] D. H. Hyers, G. Isac, and T. M. Rassias, Stability of Functional Equations in Several Variables, Progress in Nonlinear Differential Equations and their Applications, 34, Birkhäuser, Boston, Mass, USA, 1998.

[28] H. Khodaei and Th. M. Rassias, "Approximately generalized additive functions in several variables," International Journal of Nonlinear Analysis and Applications, vol. 1, no. 1, pp. 22-41, 2010.

[29] P. Nakmahachalasint, "Hyers-Ulam-Rassias and Ulam-Gavruta-Rassias stabilities of an additive functional equation in several variables," International Journal of Mathematics and Mathematical Sciences, vol. 2007, Article ID 13437, 6 pages, 2007.

[30] P. Nakmahachalasint, "On the generalized Ulam-Gavruta-Rassias stability of mixed-type linear and Euler-Lagrange-Rassias functional equations," International Journal of Mathematics and Mathematical Sciences, vol. 2007, Article ID 63239, 10 pages, 2007. 
[31] C.-G. Park and J. M. Rassias, "Hyers-Ulam stability of an Euler-Lagrange type additive mapping," International Journal of Applied Mathematics E Statistics, vol. 7, no. Fe07, pp. 112-125, 2007.

[32] C. Park, J. S. An, and J. Cui, "Isomorphisms and derivations in Lie C*-algebras," Abstract and Applied Analysis, vol. 2007, Article ID 85737, 14 pages, 2007.

[33] K. Ravi, M. Arunkumar, and J. M. Rassias, "Ulam stability for the orthogonally general EulerLagrange type functional equation," International Journal of Mathematics and Statistics, vol. 3, no. A08, pp. 36-46, 2008.

[34] M. B. Savadkouhi, M. E. Gordji, J. M. Rassias, and N. Ghobadipour, "Approximate ternary Jordan derivations on Banach ternary algebras," Journal of Mathematical Physics, vol. 50, no. 4, Article ID 042303, 9 pages, 2009.

[35] S. Shakeri, R. Saadati, and C. Park, "Stability of the quadratic functional equation in non-Archimedean $\mathcal{L}$-fuzzy normed spaces," International Journal of Nonlinear Analysis and Applications, vol. 1, no. 2, pp. 72-83, 2010.

[36] P. Găvruţa, "A generalization of the Hyers-Ulam-Rassias stability of approximately additive mappings," Journal of Mathematical Analysis and Applications, vol. 184, no. 3, pp. 431-436, 1994.

[37] D. G. Bourgin, "Approximately isometric and multiplicative transformations on continuous function rings," Duke Mathematical Journal, vol. 16, pp. 385-397, 1949.

[38] P. Šemrl, "The functional equation of multiplicative derivation is superstable on standard operator algebras," Integral Equations and Operator Theory, vol. 18, no. 1, pp. 118-122, 1994.

[39] R. Badora, “On approximate derivations," Mathematical Inequalities E Applications, vol. 9, no. 1, pp. 167-173, 2006.

[40] E. Ansari-Piri and E. Anjidani, "Superstability of generalized derivations," Journal of Inequalities and Applications, vol. 2010, Article ID 740156, 13 pages, 2010.

[41] M. Eshaghi Gordji, M. B. Ghaemi, and B. Alizadeh, "A fixed point method for perturbation of higher ring derivations in non-Archimedean Banach algebras," to appear in International Journal of Geometric Methods in Modern Physics.

[42] M. Eshaghi Gordji, "A fixed point approach to superstability of generalized derivations on nonArchimedean Banach algebras," Abstract and Applied Analysis, vol. 2011, Article ID 587097, 9 pages, 2011. 


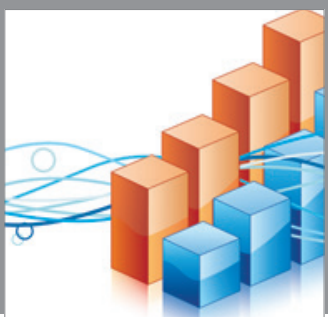

Advances in

Operations Research

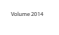

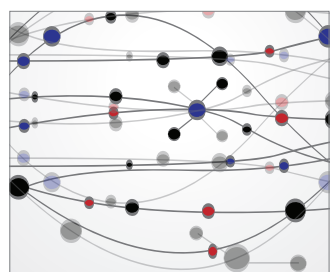

\section{The Scientific} World Journal
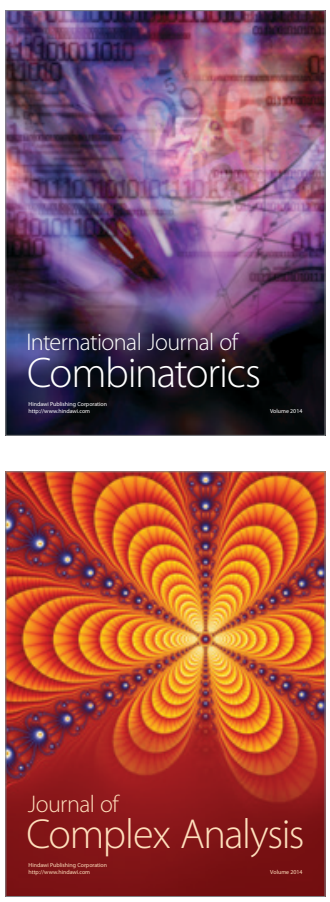

International Journal of

Mathematics and

Mathematical

Sciences
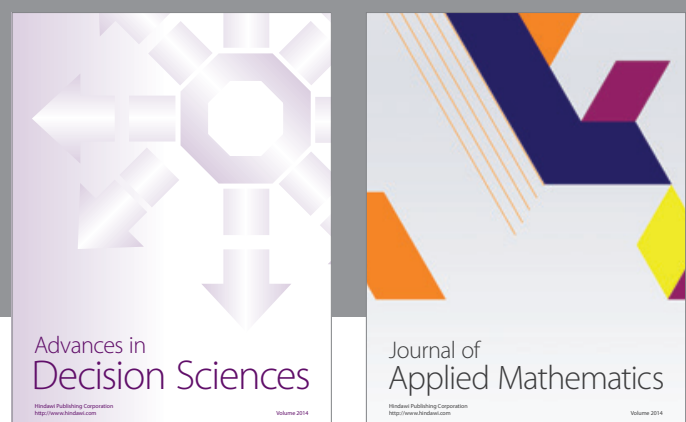

Journal of

Applied Mathematics
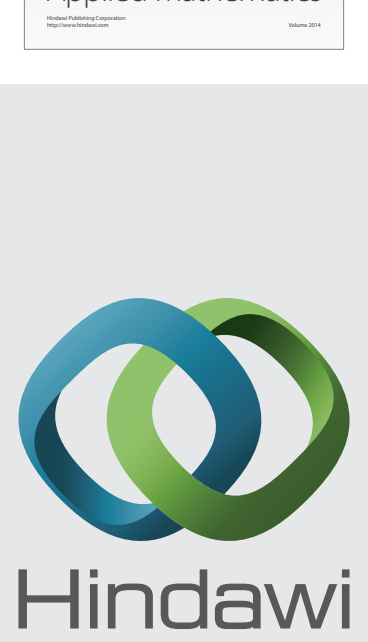

Submit your manuscripts at http://www.hindawi.com
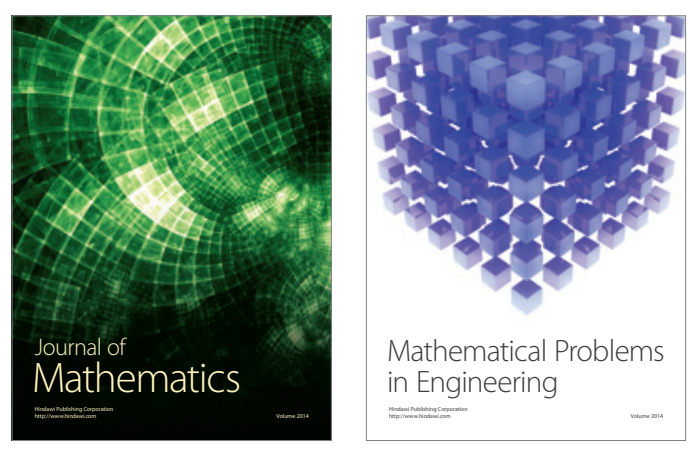

Mathematical Problems in Engineering
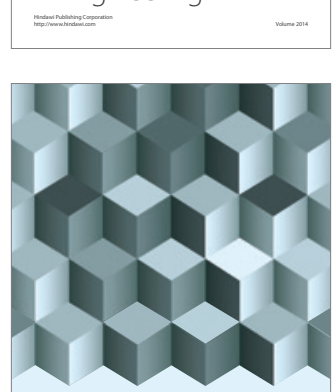

Journal of

Function Spaces
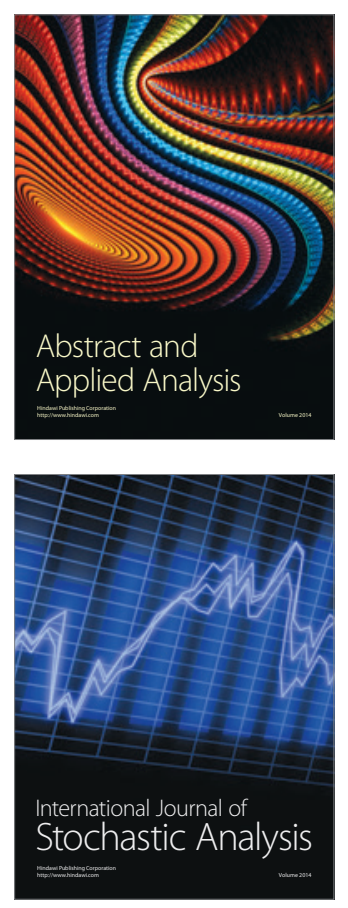

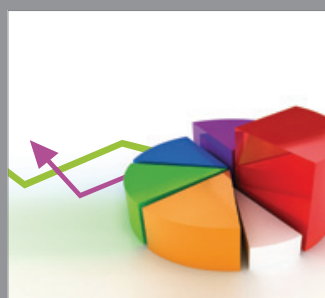

ournal of

Probability and Statistics

Promensencen
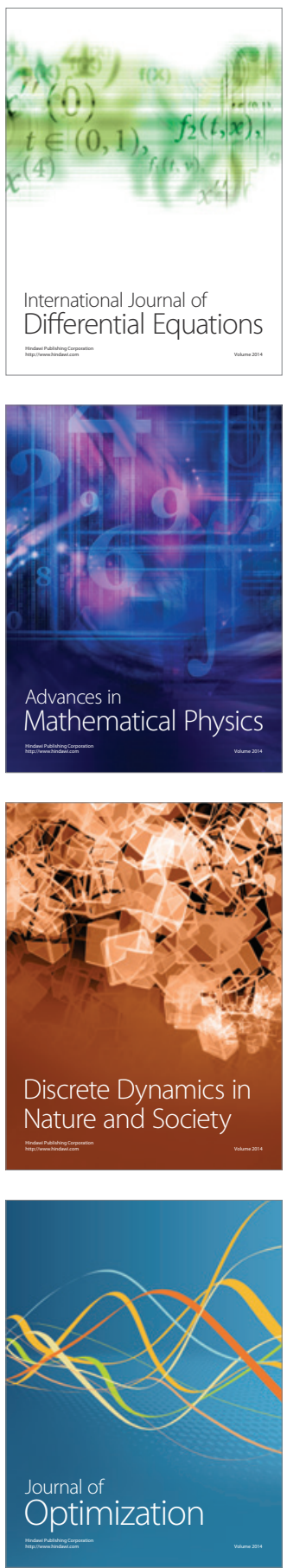